\title{
HOSPITALIZATION ISSUES IN THE PUBLIC HEALTH CARE SYSTEM: EVIDENCE FROM VIETNAM
}

\author{
Nguyen Van Phuong, Lecturer at International University \\ Email: nvphuong@hcmiu.edu.vn
}

\begin{abstract}
This paper aim to build a theory model to generalize the health care market and find the solution for the hospitalization issues in the public health care system in Vietnam. Specifically, the results indicate that increased quality improvement and reduced waiting time for patients within a hospital are able to solve if two-price tariffs are allowed to implement in the state hospitals. Additionally, by exploring the two-wave Vietnamese Household Living Standard Survey conducted in 2004 and 2006, this paper also investigates the impact of the new health insurance policy on a number of hospital visits.
\end{abstract}

Keywords: Vietnam, hospitalization issues, two-price tariffs.

\section{INTRODUCTION}

The waiting times affecting the quality of health care services are a great concern in the health care markets in many countries. Mean waiting times for nonemergent care are approximately three months in several countries, but in some cases, maximum waiting times can extend to years (Brekke et al 2008). In particular, waiting times remains a considerable issue in Vietnam where the government is pursuing a universal health care system and manages the health insurance fund. For example, on average, waiting times for heart surgery can stretch four to five months. In some circumstances, patients have to pay hospital fees in advance, yet wait for actual care for more than one year. Furthermore, waiting times for routine yearly health examinations in hospitals are the most problematic. Patients are required to arrive at the hospitals early to complete the necessary forms and then wait several hours for their appointment. Consequentially, waiting times for diagnose and treating diseases in most hospitals is causing patients and their relatives more suffering. For example, hundreds of patients arrive at the Cancer Hospital of Saigon at 3 a.m. to complete the necessary forms and then wait for hours when they begin calling names starting at 8 a.m. (nguoi-viet.com).

To reduce waiting times, many policymakers propose more competitive healthcare markets to improve the quality of services and to decrease waiting lists. Many studies show that market competition on health care services has a close relationship with the quality of service and waiting times (Olivella (2002); Siciliani and Hurst ( 2005), Siciliani (2005); Barros and Olivella (2005); Siciliani (2007), Brekke et al (2008)); and Felder (2008)). Generally speaking, recent literatures have considered waiting times in the context of excess market demand. It is more likely to assume that the quality of treatment is an identical factor for each patient at the same hospital. In other 
words, the quality of health care services and waiting times are considered in the context of market competition effects. The waiting times could improve if the quality of a particular hospital is better than another in a competitive market. Unlike previous studies, this paper illustrates the importance of quality improvement plays in relationship to decreased waiting times and fewer hospital visits. Currently, however, to achieve such benefits, the patients need to pay above and beyond what insurance will cover for services.

In empirical studies, the paper investigates the effects of the new health insurance policy on the hospital visits. By modifying the classical demand for healthcare services following the Grossman model, the estimated results are consistent with previous studies as following: 1> an increase in price of hospital charges lead to lower demand for healthcare services; $2>$ the higher education level a person achieves, the less likely she/he is to visit the hospital; $3>$ The symptom of Moral hazard is more likely to appear. Furthermore, the paper also implements another econometric method to robust test by using the Poison model. The estimated coefficients have attained more insightful and empowered results.

This study employs data taken from the two-wave Vietnamese Household Living Standard Survey conducted in 2004 and 2006 by the General Statistical Office of Vietnam. After unifying identical notation variables of both waves, the two surveys were merged to form the cross sectional data for two years with 79,509 observations. Additionally, we also create a matching program to provide balanced panel data with 26,508 observations. The data provides detailed information of a nationwide sample of Vietnam based on the characteristics of current household living standards; all individuals in a family, employment status including careers and industries, health and disability covering health status and insurance schemes. Since the data covers a two-year period, analysis considers the effect of the new health insurance policy (which took effected on 07/01/2005) on the demand for hospital visits.

The paper is structured as follows: the next Section provides a brief review of the related literature. Section 3 summarizes key features of the Vietnamese health care system. The model for this research is generalized in Section 4, while in Section 5 a description of data is presented. In Section 6, empirical strategies and a discussion of empirical specification are briefly presented. Finally, Section 7 makes some final remarks.

\section{Literature review}

Waiting times for health care treatment are a significant health care policy concern not only in developed countries but also in developing countries. For instance, in the United Kingdom and Finland, average wait times for hip or knee replacement, cataract surgery or varicose veins can be as long as seven to eight months (Siciliani, 2007). To reduce waiting times, the policymakers argue that increased competition and patient alternatives can decrease waiting times by encouraging hospitals to provide better health services to attract more patients and maximize revenues (Siciliani and Hurst, 2005). In theoretical studies, many authors construct models to explain the relationship between competition and waiting times in hospital markets. For example, Olivalla (2002); Barros and Olivella (2005); Sicilian (2005) and several other studies have constructed theoretical models in which the hospitals are assumed to be local monopolists or duopolistic market structure. In the recent study, Brekke et 
al (2008) expand on the previous models by introducing the geographical location variables and allowing competition between local monopolists. As a result, they show that local hospitals compete to decrease waiting times whenever the competitive demand market is sufficiently small. In general, most of the existing literature is focused on waiting times in the context of the excess demand market for health care services.

Unlike previous studies, this study constructs a general model to show that quality improvement might implement within the hospital. The higher quality of health care services in an internal hospital, the less waiting times and the fewer hospital visits patients could benefit from hospitalization. However, to achieve the benefits, patients need to pay extra charges for services on demand.

The number of hospital visits is considered as the demand for healthcare services. Pohlmeier and Ulrich (1995) have developed the Grossman model to investigate the determinantfactors affecting on the demand for medical services. They show that independent variables such as a price vector for hospital services, the latent variable "health status", the wage rate, the time trend, the level of education and the living environment effects have strongly influenced on the demand for medical services. By modifying their model as well as implementing another econometric method of the Poisson model, the paper finds insightful results consistent with previous studies.

The Vietnamese healthcare system

Currently, there are two types of health insurance in Vietnam. The first one is compulsory for those who have labor contracts at least three months in length. The premium rate is three percent of the salary written in the labor contract per month (base salary), of which the employer pays two percent and the employee percentage is one percent ${ }^{1}$. Moreover, this type of health insurance also includes those who are retired or are receiving social benefits. This group receives a health insurance card without paying the premium rate.

The second option is voluntary health insurance, pupils and students are primary participants. Although it is called voluntary health insurance, almost all pupils and students are required to pay health insurance fees at the beginning of school year, except for those who are living in poor families. According to the circular number 06/2007/TTLT-BYT-BTC issued on 03/30/2007 from the Ministry of Health and Ministry of Finance, the premium fees for pupils and students in urban areas ranges from 60,000 to $120,000 \mathrm{VND} /$ person/ year, and in rural areas, it ranges 50,000 $100,000 \mathrm{VND} /$ person/year. Meanwhile the annually premium fees for an adult living in urban and in rural areas are 160,000 $-320,000 \mathrm{VND}$, and $120,000-240,000$ VND, respectively. Until now, the adults without formal employment have not been interested in participating in the voluntary insurance policy even though the current premium levels are very low as compared to the real hospital charges when they require medical care. On one hand, these individuals are concerned about the low quality services provided by local state health care providers. On the other hand, they are not confident in the real benefits of health care insurance due to complicated procedures to obtain reimbursement and the cap policy for treatment as individuals can only be treated with the medicines and technical diagnoses on specific approval lists. 


\section{Model}

We generalize the model of hospitalization reflecting the health care system and health insurance policy in Vietnam. Whenever a patient gets a severe condition, she/he is looking forward to seeking a professional treatment at a hospital or emergency room. Currently, in order to receive full benefits of the health insurance policy in Vietnam, an insured patient is required to be treated at a primary hospital registered with the health insurance company. Since the government is pursuing the universal health system, the premium rate has been built at a very low rate to encourage the voluntary buyers. The insurance premium $(\varepsilon)$ is a small amount. The benefit function $b(h, q) b(h, q)$ is a strictly concave function, $\boldsymbol{h} \boldsymbol{h}$ represents a number of treatments at a hospital, and $q$ denotes the quality of remedy. The utility function of an insured patient when ill and treated at a hospital follows:

$$
\begin{aligned}
& U\left(Y-\varepsilon+b(h, q)(1-g(w))-e\left(h, p_{1}, p_{0}\right)-s\right) \\
& U\left(Y-\varepsilon+b(h, q)(1-g(w))-h p_{1}+h p_{0}-s\right)
\end{aligned}
$$

Where $Y$ denotesindividualincome, $p_{1}$ $p_{1}$ represents the total health expenditures; and $p_{0} p_{0}$ represents the deductible cost of using the baseline medical service in the cap regulation for each treatment. Depending upon the number of medical services per hospital treatment, $p_{0} p_{0}$ is fully or partial reimbursable. Meanwhile $\mathrm{g}(\mathrm{w})$ is a discount function of waiting times $(w)$ if baseline services are required. $\left(0<g(w)<1, \quad g_{w}(w)>0, g_{w w}(w)<0\right)$, $s$ is an opportunity cost during treatment, $e\left(\boldsymbol{h}, p_{1}, p_{0}\right) e\left(\boldsymbol{h}, p_{1}, p_{0}\right)$ is a hospital fee function assumed linear relationships between $\mathrm{h}$ and $p_{1}$, and $p_{0} p_{1}$, and $p_{0}$, and OoP (OoP) payment per treatment is the difference between $p_{1}$ and $p_{0} p_{1}$ and $p_{0}$.

When an insured patient uses baseline services, she/he will have to wait for a certain time to receive the treatment with the benefit being reduced correspondingly with the interactive term of discount function $(b(h, q) * g(w) b(h, q) * g(w))$. The more baseline services used, the longer waiting time and fewer benefits in terms of effective treatment the insured patient may receive. The insured patient chooses $h$ to maximize $U().($.$) . The necessary$ and sufficient first order condition for a local maximum $U().($.$) is to take a partial$ derivative with respect to $h$ of Equation (1), we yield the following result:

$$
\begin{aligned}
b_{h}(h, q)(1-g(w))-p_{1}+p_{0}=0 \\
b_{h}(h, q)(1-g(w))-p_{1}+p_{0}=0
\end{aligned}
$$

The result implies that the insured patient chooses $\boldsymbol{h} \boldsymbol{h}$ to set the marginal benefit of treatment equal to the OoP health expenditure per treatment $\left(p_{1} 1-p_{1} 0\right)$ $\left.p_{1} 1-p_{1} 0\right)$. From Equation (2), the result derived:

$$
\frac{\partial p_{\mathbf{1}}}{\partial \boldsymbol{h}}-\frac{\partial p_{0}}{\partial \boldsymbol{h}}=b_{h \boldsymbol{h}}(h, q)(1-g(w))<\mathbf{0}
$$

Considering the sign of each item in the left hand side of Equation (3), the first item is negative. This indicates that the more hospital treatments, the higher OoP amount an insured patient has to pay. Meanwhile, the second item is positive because the more hospital treatments, the more reimbursement an insured patient might receive. It is worth noting that since the new law of extending reimbursable amounts for the insured persons took effect, many new and current enrollees now have an incentive to visit state hospitals. Moreover, the new regulation also sets the maximum amount that the insured is allowed to be reimbursable per hospitalization treatment. This would lead to increate in a number of visits to avoid consuming over the limited amounts.

We now analyze the hospital's utility expected from treatment. The main revenues of a state hospital include 1> 
the subsidized capital for physical and infrastructural investment $(\mathrm{T})$ from the government to maintain the public health care system, $2>$ the health care service fees per treatment collected directly from patients' OoP service fees $\left(p_{1} 1-p_{1} 0\right)$ $\left.p_{1} 1-p_{1} 0\right)$, and $3>$ the reimbursable amounts $\left.\left(p_{1} 0\right) p_{1} 0\right)$ per treatment received from the health insurance fund. The main expenses that a state hospital are responsible for are fixed costs $(\mathrm{F})$, marginal cost of baseline services $c\left(q_{0}\right) c\left(q_{0}\right)$, and marginal cost of services on demand and improvement in quality of services $c(q)$ $c(q)$. To achieve a universal healthcare system, the tariffs of baseline services are strictly controlled to guarantee that the health fee of baseline services is sufficient to cover its marginal cost (i.e $p_{0}-c\left(q_{0}\right)$ $\left.p_{0}-c\left(q_{0}\right)=0\right)$. The hospital's utility can be written as:

$$
V\left(T+h *\left(p_{1}-p_{0}\right)+h * p_{0}-F-h * c\left(q_{0}\right)-h * c(q)\right)
$$

The hospital chooses $\mathrm{q}$ to maximize its revenues less costs and the patient subsequent choice of $h$ to maximize her/ his utility to form the subgame-perfect equilibrium as:

$$
\max _{q} T+h *\left(p_{1}-p_{0}\right)-F-h * c(q)
$$

subject to $p_{1}-p_{0}=b_{h}(h, q)(1-g(w))$

$$
q=\left[\frac{\propto(1-\propto)}{2} h^{\alpha-1}(1-g(w))\right]^{\frac{1}{\alpha+1}}
$$

Most of the previous studies about the relationship between the quality of health services and waiting times have focused on the external effects of competition in hospital markets. Unlike previous studies, the results in Equation (5) illustrates that the more quality improvements are made in an internal hospital, there is a decline in waiting times and the fewer hospital treatments. However, the quality of health care services might improve in severe excess demand conditions if the two price tariffs for the health care markets including the high price for services on demand and low price for baseline services should be allowed to perform simultaneously.

\section{Data}

This paper explores the two-wave Vietnam Household Living Standard
We now consider the benefit function $(b(\boldsymbol{h}, q) b(\boldsymbol{h}, q)$ and marginal cost $c(q) c(q)$ are able to be presented as specific functions to solve the above maximum problem. We are assuming that $b(h, q)=h^{\alpha} q^{1-\alpha}(\alpha<1)$ and $c(q)=q^{2}$. $b(h, q)=h^{\alpha} q^{1-\alpha}(\alpha<1)$ and $c(q)=q^{2}$.

Solving the maximum problems of Equation (4), the results yields:

$\frac{d w}{d q}<0, \quad$ and $\frac{d \boldsymbol{h}}{d q}<\mathbf{0}$

Survey conducted in 2004 and 2006 by the General Statistical Office of Vietnam. The survey in 2004 interviewed 9,300 households including 40,438 observations. The survey in 2006 interviewed 9,189 households including 39,071 observations. Since the notation variables of both datasets are not unified, we have to unify the identical notation variables before merging both datasets to conduct the cross section data for two years with 79,509 observations. Otherwise, the repeated household surveys in two periods are randomly selected and account for approximately 30 percent of total samples (based on our matching program). Nevertheless, the personal identification numbers of both surveys to conduct the panel data were unavailable. In evaluating location (province, district, 
ward, cluster), full name, gender, year of birth, month of birth and day of birth if available, we create a matching program to conduct the balanced panel data of 2 twoyear surveys with 26,508 observations. In addition, all financial variables are deflated by the growth rate of consumer price index of year 2004 .

Exploring the two-wave data of 2004 and 2006 enables an analysis of the impact of the new Decree No.63/2005/ND-CP expanding more benefits for the insured patients taking effect on $07 / 01 / 2005$ on the hospitalization rate and OoP payment of the insured and uninsured patients.

The descriptive statistics for key variables are presented in Table 1.

Table 1: Descriptive statistics of key variables

\begin{tabular}{|c|c|c|c|c|}
\hline Variables & Description of variables & Obs & Mean & $\begin{array}{l}\text { Standard } \\
\text { Deviation }\end{array}$ \\
\hline ln_total & Natural logarithm of total hospital visits & 23450 & 0.6321 & 0.7485 \\
\hline ln_Out_P & Natural logarithm of out-patient visits & 20929 & 0.6640 & 0.7587 \\
\hline ln_In_P & Natural logarithm of in-patient visits & 3762 & 0.1305 & 0.3607 \\
\hline \multirow{3}{*}{$\begin{array}{l}\text { ln_OoP_per total visit } \\
\text { ln_OoP per Out_P visit }\end{array}$} & Natural logarithm of total OoP per visit & 20813 & 4.1918 & 1.5634 \\
\hline & & & & \\
\hline & Natural logarithm of OoP per out-patient visit & 18302 & 3.8745 & 1.3349 \\
\hline \multicolumn{5}{|l|}{ ln_OoP per In_P visit } \\
\hline & Natural logarithm of OoP per in-patient visit & 3564 & 6.1114 & 1.5167 \\
\hline age & Age in years & 79509 & 30.4214 & 20.2016 \\
\hline edu & Number of years of education & 79509 & 6.5531 & 4.0312 \\
\hline urban & $1=$ urban; other $=0$ & 79509 & 0.2385 & 0.4262 \\
\hline Insu & $1=$ have health insurance; other $=0$ & 79509 & 0.4709 & 0.4992 \\
\hline ln_inc_pc & Natural logarithm of Household Income per capita & 79493 & 6.1214 & 0.7001 \\
\hline water_well & $1=$ use water from well; other $=0$ & 79509 & 0.2164 & 0.4118 \\
\hline self_employ & $1=$ self employment; other $=0$ & 79509 & 0.4735 & 0.4993 \\
\hline wage_employ & $1=$ wage employment; other $=0$ & 79509 & 0.2363 & 0.4248 \\
\hline unemploy & $1=$ unemployment; other $=0$ & 79509 & 0.3380 & 0.4730 \\
\hline disabled & $1=$ disabled; other $=0$ & 79509 & 0.0069 & 0.0826 \\
\hline gender & $0=$ female $; 1=$ male & 79509 & 0.4929 & 0.5000 \\
\hline Marital Status & $0=$ single $; 1=$ married & 79509 & 0.4528 & 0.4978 \\
\hline
\end{tabular}

Source: Two Surveys of Vietnamese Household Living Standard conducted in 2004 and 2006

\section{Empirical Strategies}

We consider the impact of new health insurance policy on the demand for health care services. Each type of hospital visits is considered as the primary dependent variables and the data are taken from the household surveys instead of hospital discharges, so that a number of considerable observations are not used whenever dependent variables are measured directly a number of hospital visits. We include 64 provincial fixed effects to capture any permanent differences across provinces that might contribute to their level of hospitalization rates. To test such an impact, we modify the classical demand function for healthcare services that emerges from the Grossman model as following (see Pohlmeier \& Ulrich, 1995 for more details): 


$$
\begin{aligned}
\ln \left(y_{i j t}\right)=\beta_{0}+ & \beta_{1} \ln \left(P_{i t}\right)+\beta_{\mathbf{2}} I n s_{i j t}+\beta_{\mathbf{3}} \ln \left(\operatorname{Incpc}_{i j t}\right)+\beta_{\mathbf{4}} a g e_{i}+\beta_{\mathbf{5}} y_{2006} \\
& +\beta_{\mathbf{6}} E n v_{i j t}+\beta_{7} E d u_{i j t}+\beta_{\mathbf{8}} Z_{i j t}+\beta_{\mathbf{9}} l o c_{t}+u_{i j t}
\end{aligned}
$$

Where $i$ denotes individual, $j$ indexes for location, $t$ denotes time, $\mathrm{y}$ is measured as a number of total hospital visits (it also denotes other dependent variables of out-patient visits), $P$ denotes a price vector for hospital services per visit, Ins is a dummy variable for health insurance status, Incpc is income per capita, y_2006 is a dummy variable of year 2006, Env is a vector of environment effects, $E d u$ is years of education, $Z$ is a vector of social, demographic, biological control variables for individual, loc indexes for location at district level, $t$ is time trend, $u$ is an unobserved error component. It is worth mentioning that a vector price of healthcare services per hospital visit measured as OoP expenditures per hospital visit is one of the key independent variables. It enables us to analyze the effects of changes in health care expenditures on the demand.

The environment variable is measured as the sanitary level of the living environment around a household that could have an effect on individual's health status. More precisely, it is measured as a dummy variable of unsanitary water use, and equal to one if household directly use water taken from a well, and zero otherwise. The last independent vector of control variables is $Z$. It includes a full set of dummy variables such as self employed, employment, unemployment, disabled, gender, married, child/children below age 16 .

We have already implemented the empirical test of Equation (6) by using OLS method ${ }^{2}$. Here, we would like to present another econometric method by using the Poisson regression.

Indeed, the datasets of hospital visits can be considered as count data. Therefore, we might set up a Poisson model to implement an empirical test. The Poisson model can enable explanation related to the number of hospital visits during a given time period. Clearly, the outcomes are recorded as count data in which the zero value might account substantially for the population. To avoid the overdispersion, two methods are evaluated simultaneously. First, in processing data, the model considers a natural logarithm of OoP expenditures per hospital visit as one of the key independent variables. By doing so, it will drop all zero value in dependent variables. Second, to account for the nonindependence between observations from the same person, a random intercept model to handle the over-dispersion is introduced. Finally, the empirical model is rearranged as the Poisson regression model by the following equations:

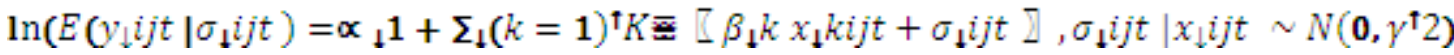

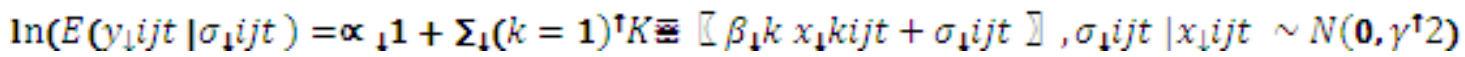

Where $y_{i j t} y_{i j t}$ denotes the total number of hospital visits of a patient (i) in location $(\mathrm{j})$ during the period time $(\mathrm{t})$, $\alpha_{1} \propto_{1}$ indicates an intercept, $x_{k i j t} x_{k i j t}$ is a k-dimensional vector of explanatory variables and $\beta_{k} \beta_{k}$ is a $\mathrm{k}$-dimensional vector of parameters. While $\sigma_{i j t} \sigma_{i j t}$ is an error term for the $i^{\text {th }}$ patient that follows a log-gamma distribution with mean zero, reflecting unobserved heterogeneity between individuals. 
To analyze the data using Poisson regression with random effects for both datasets, an xtpoisson procedure in the STATA statistical package was employed. Since personal numbers are not identical in the cross section dataset, the location at district level as the group variable for random effects was utilized. By doing so, we can fit separate constants for each location and crediting the regression model with explanatory power that enables explanation of the variance over time relative to the mean rates of a number of hospital visits for each location. In the panel data, we use personal identification numbers $^{3}$ for random effects. All independent variables and a vector control of variables similar to Equation (6) are the same in the Poisson regression model. The only one difference is the dependent variable that is measured as count data.

Table 2 summarizes the results of a Poisson regression from Equation (7) with location as the group variable for random effects from Columns (1) to $(2)^{4}$. Meanwhile, Columns (3) to (4) presents the estimated results with personal identification numbers for random effects of the 2-year panel dataset. The empirical test of Equation (7) with individual fixed effect was implemented resulting in numerous statistically insignificant results due to time-invariant characteristics of control variables. Therefore, the results are not reported in the section.

Table 2: The effects of OoP money and the new health policy on the hospitalizationRandom-effects Poisson model

\begin{tabular}{|c|c|c|c|c|}
\hline & (1) & (2) & (3) & (4) \\
\hline & Total & Out_P & Total & \\
\hline \multirow[t]{2}{*}{$\ln (\mathrm{OoP})$} & $-0.1282 * * *$ & $-0.1178 * * *$ & $-0.1424 * * *$ & $-0.1299 * * *$ \\
\hline & $(0.0043)$ & $(0.0052)$ & $(0.0093)$ & $(0.0116)$ \\
\hline \multirow[t]{2}{*}{ y2006* $\ln (\mathrm{OoP})$} & $-0.0330 * * *$ & $-0.0527 * * *$ & $-0.0457 * * *$ & $-0.0731 * * *$ \\
\hline & $(0.0061)$ & $(0.0071)$ & $(0.0135)$ & (0.0159) \\
\hline y2006* $\ln (\mathrm{OoP}) *$ & $0.0163 * * *$ & 0.0068 & $0.0240 * * *$ & $0.0168^{*}$ \\
\hline Hlth_insu & $(0.0040)$ & $(0.0046)$ & $(0.0087)$ & $(0.0101)$ \\
\hline \multirow[t]{2}{*}{ y2006 } & $0.1393 * * *$ & $0.2071 * * *$ & $0.1282 * *$ & $0.2152 * * *$ \\
\hline & $(0.0246)$ & $(0.0274)$ & $(0.0549)$ & $(0.0619)$ \\
\hline \multirow[t]{2}{*}{ hlth_insu } & $-0.0448 * * *$ & $-0.0306^{* *}$ & $-0.1167 * * *$ & $-0.1018 * * *$ \\
\hline & $(0.0127)$ & $(0.0135)$ & $(0.0287)$ & $(0.0313)$ \\
\hline \multirow{2}{*}{ ln_inc_pc } & $0.0803 * * *$ & $0.0804 * * *$ & $0.1453 * * *$ & $0.1404 * * *$ \\
\hline & $(0.0078)$ & $(0.0082)$ & $(0.0176)$ & $(0.0190)$ \\
\hline \multirow[t]{2}{*}{ water_well } & $0.0350 * * *$ & $0.0467 * * *$ & 0.0115 & 0.0191 \\
\hline & $(0.0134)$ & $(0.0140)$ & $(0.0271)$ & $(0.0290)$ \\
\hline
\end{tabular}

\footnotetext{
${ }^{3}$ These identification numbers are created from our matching program.

${ }^{4}$ We also perform a robust test for district fixed effects and the results are almost similar.
} 


\begin{tabular}{lcccc}
\hline & & & & \\
& $(1)$ & $(2)$ & $(3)$ & $(4)$ \\
& Total & Out_P & Total & Out_P \\
\hline \multirow{2}{*}{ edu_years } & & & & \\
& $-0.0136^{* * *}$ & $-0.0146^{* * *}$ & $-0.0251^{* * *}$ & $-0.0272^{* * *}$ \\
& $(0.0015)$ & $(0.0015)$ & $(0.0036)$ & $(0.0039)$ \\
\hline $\mathrm{N}$ & 20785 & 18277 & 6721 & 5929
\end{tabular}

The results of a Poisson regression with location as the group variable for random effects from column 1 to 2 . While Column (3) to (4) presents the estimated results with individual identify number for random effects of the 2-year panel dataset. Standard errors in parentheses. * significant at $10 \% ; * *$ significant at $5 \% ; * * *$ significant at $1 \%$

Considering some remarkable results presented in Table 2 , from theory of point of view, an increase in price of medical services will lead to lower demand for health care services. These results are consistent with the theory. The coefficients of out-of-pocket per hospital visit have a negative relationship with the hospitalization visits and are statistically significant at the $1 \%$ level.

Additional results also included the y2006 dummy variable for the survey conducted in 2006 that is statistically significant and indicates a positive relationship with demand for the total number of hospital visits and out-patient visits. It implies that the new policy extending many benefits for the insured leads to increase demand for primary healthcare services. The new policy is more likely to attract new enrollees and current insured persons to maximize the utility of medical services for the insured. However, an increase hospital visits, the larger amount of OoP expenditures will occur because of many indirect costs generated such as transportation, food, waiting time, and especially the "informal payments" for hospital staff. The estimated results could explain, at least in part, these public health care hurdles. The result of interaction term between y2006 and $\ln (\mathrm{OoP})$ is a negative association with total number of hospital visits and out-patient visit.

The main objective of the new policy could achieve some degrees of beneficiaries. As a result of the increase in the cap regulation, the insured patient is able to reduce some direct healthcare fees which in turn increased the demand for primary healthcare services since 2005 . In particular, the coefficients of the interaction $(\mathrm{y} 2006 * \ln (\mathrm{OoP}) *$ hlth_insu) are positively associated with the total hospital visits and are statistically significant. The signal of Moral Hazard is more likely to appear when the insured patients increase outpatient visits to take advantages of the new benefits for the enrollees since 2005 .

The next result presented is the level of education. The higher education level person attains, the less likely they are to visit the hospital and use primary health care services. Again, the well educated persons are more likely to pay attention to the health information to prevent sickness rather than concerning the related input of demand function for the health treatment. This result is also consistent with many previous studies (Pohlmeier and Ulrich, 1995, Schellhorn, 2001, Card et al, 2008).

The result in Table 3 shows that the individuals with a higher income 
are more likely to prefer higher quality healthcare services. However, the results from this research are not completely consistent with several previous findings. For example, Riphahn et al (2003) find that a positive relationship only occurs in demand of females for additional hospital services. Meanwhile, Schellhorn (2001) shows that income has insignificant effect on physician service utilization. However, it is worth recalling that many previous studies regarding health insurance and demand for medical services have used the dataset of developed countries where private health insurance is a primary option for high income families. Therefore, the level of income would play an important role in rationalizing the health insurance plans ex ante, rather than directly affecting the demand for health treatment ex post. Overall, according to a particular plan, the insured will determine demand for primary care and hospital visits. In contrast with this research where the government manages the health insurance fund and offers only a basic coverage for all insured persons. Therefore, only the higher income patients can afford to pay for additional services on demand at hospitals as well as access other expensive medical services.

The living environment around a household has played an essential role in human health status. Here, water resource is considered as one of the key variables to test the impact of living environment on demand of individual for medical services. The quality of water resources has a significant impact on resident health status. Therefore, households using direct water taken from a well without filtering or boiling could face a high risk of deteriorated health and increased incidents of minor diseases such as cholera, diarrhea, vomiting, gastroenteritis and so forth. Our results demonstrate that an increase in using untreated water taken from a well is associated with an increase in the number of total hospital visits and out-patient visits with statistically significant at the $1 \%$ level.

\section{CONCLUSION}

We show that with increased quality improvement of health care services within a hospital, waiting times and quality of health services will improve whenever the state hospitals are enable to implement two price systems including the high price for services on demand and low price for baseline services on demand.

In empirical test, our results are consistent with previous studies. The factors such as the out-of-pocket payment, level of education, environment effect are playing significant role in effecting on a number of hospital visits. Specifically, we find the symptom that is commonly associated with moral hazard. This issue needs further investigate in future studies. 


\section{REFERENCES}

Barros, Pedro Pita and Pau Ovivella. (2005). Waiting Lists and Patient Selection. Journal of Economics \& Management Strategy , 24, 623-646.

Brekke, Kurt R., Luigi Siciliani, Odd Rune Straume. (2008). Competition and waiting times in hospital markets. Journal of Public Economics, 92, 1607-1628.

David Card, Carlos Dobkin, and Nicole Maestas. (2008). The Impact of Nearly Universal Insurance Coverage on Health Care Utilization: Evidence from Medicare. American Economic Review , 98, 2242-2258.

Olivella, P. (2002). Shifting public-health-sector waiting lists to the private sector. European Journal of Political Economy, 19, 103-132.

Pohlmeier, Winfried and Volker Ulrich. (1995). An Econometric Model of the TwoPart Decisionmaking Process in the Demand for Health Care. The Journal of Human Resources , 30, 339-361.

Schellhorn, M. (2001). The Effect of Variable Health Insurance Deductibles on the Demand for Physican Visits. Econometrics and Health Economics , 10, 441-456.

Sicilian L and Hurst J. (2005). Tackling excessive waiting times for elective surgery: a comparison of policies in twelve OECD countries. Health Policy, 72, 201-215.

Siciliani, L. (2005). Does more choice reduce waiting times? Health Economics, 14, 17-23.

Siciliani, L. (2007). Optimal Contracts for health services in the presence of waiting times and asymmetric information. The Berkeley Electronic Press . 